\title{
Ultrasound Imaging of Liver Tumors - Current Clinical Applications
}

\author{
R. Badea ${ }^{1}$ and Simona Ioanitescu ${ }^{2}$ \\ ${ }^{1}$ Ultrasound Dept., Institute of Gastroenterology and Hepatology, \\ Univ. of Medicine \& Pharmacy "Iuliu Hatieganu" Cluj-Napoca \\ ${ }^{2}$ Center of Internal Medicine, Fundeni Clinical Institute, Bucharest
}

Romania

\section{Introduction}

\subsection{Definition of ultrasonographic method}

Ultrasound exploration (ultrasonography) is a very common diagnostic method. It is part of imaging procedures, without using ionizing radiations but ultrasounds (US) with usual frequencies of 2 - 5 - $12 \mathrm{MHz}$. Ultrasounds cross biological environments and are reflected at the demarcation limit between structures of different consistencies. The current procedure of ultrasound examination called "scanning" is based on the analysis of every plane from a region of interest in the human body. Each plane contains a high number of points with different brightness (within the limit of the gray scale used by the equipment) and their sum makes a defining "echostructure" for each organ. Ultrasound diagnosis is based on changes in tissue density due to pathological changes, resulting in echostructure transformation. Ultrasonography is an anatomical, hemodynamic and functional exploration.

\subsection{Brief history}

Since the 1940s there have been pioneers (physicians, biologists, physicists) who intuited that acoustic energy, at that time used only in war industry to detect submarines, could have applications in medical diagnosis. The first ultrasound images contained information about the density of tissues displayed along an axis ("A- mode" ultrasound). Later, "sectional" ultrasound was invented, which detailed echoes in a plane ("B-mode" ultrasound with applications in obstetrics). In the '60s the procedure had an accelerated development by diversifying examination techniques, identifying more clinical applications and increasing access to a large number of specialties. In the '70s clinical specialties such as obstetrics and cardiology "claimed" the method, using as the main argument its clinical character arising from the direct relationship between the examining physician and the patient. After the ' $90 \mathrm{~s}$, emergency physicians requested the presence of miniature, portable ultrasound equipment in the emergency room proving that ultrasound, using accordingly well defined algorithms, contributed to saving lives. Today, ultrasonography is used by clinicians in more than $50 \%$ of cases. Although there is still an ongoing "battle" between clinicians and radiologists for the monopoly over the ultrasound method, the final outcome is an exceptional dynamic of 
the method, considered today the most common diagnostic imaging procedure in the world (Derchi \& Claudon, 2009). In the near future the ultrasound examination will be unrestrictedly generalized with the introduction of the procedure as basic training for medical students, as part of clinical examination.

\subsection{Advantages and disadvantages of the ultrasound method}

Ultrasound is useful for the practitioner as a first imaging procedure in direct correlation to the clinical examination. The method has a very good cost/quality ratio, the image is very accurate and precise and the information has a dynamic character ("real time imaging"). It is important to note that ultrasound has its limitations which the examining physician must take into account. Thus, the ultrasound image contains a number of artifacts and is mainly limited by phenomena such as US attenuation related to distance and density. Ultrasound is an operator-dependent method, thus its reproducibility is reduced.

\subsection{Ultrasound techniques and procedures}

The principles of ultrasonography are complex. The US picture is generated by ultrasound penetration into the human body. US are reflected as echoes and converted by the transducer into signals. The ultrasound image is multimodal. There are multiple US procedures systematized into "clinical applications" and the information obtained is tissular ("morphometric" type) and vascular ("hemodynamic "type). Finally, US examination is a "real time" procedure which reflects the movements of the organs. It is mandatory to connect the US information to the clinical and functional-biochemical data in order to obtain the final diagnosis.

\subsubsection{Tissue investigation}

The conventional US currently used in practice is called " $2 \mathrm{D}$, gray scale" sonography. The technique is based on US property with frequencies $>20 \mathrm{kHz}$ and constant acoustic power to cross tissues with an average speed of $1540 \mathrm{~cm} / \mathrm{sec}$. US are returned with different acoustic power at a variable timeframe depending on the acoustic density of the crossed environment and on the position of the reflecting element. There is a proportional relationship between the intensity of the echo and the density of a crossed biological environment; therefore, ultrasound is a noninvasive tissue density assessment procedure. There are two categories of echoes according to their frequency: basic (similar to the incident beam, i.e. $3 \mathrm{MHz}$ ) and "harmonic" (multiples of the emission frequency, i.e. $6 \mathrm{MHz}$ ). Harmonic echoes arise from the non-linear vibration of the tissues. Due to their high frequency, the result is a high quality image. Harmonic ultrasound technique ("coded harmonics" or "tissue harmonic imaging," THI) combined with the pulse inversion procedure ("pulse inversion harmonic imaging") allows to obtain information regardless of the depth where the region of interest lies and is available on most commercial equipment (Choudhry et al, 2000).

Image resolution (minimum size at which a reflected structure is distinctly shown on the screen) is essential for tumor detection. The number of crystals included into the transducer and the nominal frequency of the ultrasound beam (the higher the frequency the better the resolution) also contribute to the ultrasound image quality. The gain of echoes ("gain" 
function) and the time compensation of echoes gain ("time gain compensation" function) measured in decibels, image depth and the number of focuses, echoes acquisition and their representation rate are other elements that allow information rendering with the same quality at an approximate ultrasound penetration of 20 to $25 \mathrm{~cm}$. All these phenomena contribute to image the tissue echostructure. The sonography has a very good capacity to discriminate lesions depending on their consistency (a parenchymal cyst is detectable at a 2$3 \mathrm{~mm}$ size!; a solid nodule is distinctly shown at sizes of $5-6 \mathrm{~mm}$ ). In addition, the method allows accurate assessment of the lesion size and the evaluation of organ motility (gastrointestinal tract, heart, main vessels).

The ultrasound image is planar, two-dimensional (2D ultrasound). In recent years equipments have been developed, allowing 3D reconstruction of ultrasound images. The resulting images are not planes but volumes that can be static (3D) or dynamic (4D). 3D/4D exploration has recognized obstetrical applications but other areas of application of this method have been identified in recent years, mainly in oncology. 3D/4D ultrasound provides accurate information in connection with the space, shape, size and tumors texture. This procedure may be useful to measure the real tumor volume as well as the healthy surrounding parenchyma which should to be removed in case of surgery. Combining this procedure with intratumoral circulation assessment methods allows more accurate diagnosis of the tumor nature (Badea et al, 2007).

\subsubsection{Blood flow and microcirculation investigation}

Blood flow can be explored by ultrasound using various techniques. Some are based on Doppler principle (or derivatives) and others on the use of intravenous contrast agents (CA).

\subsubsection{Doppler ultrasound}

It is an established method for the assessment of blood flow based on the frequency variation of a US beam hitting a target (in our case, groups of red blood cells) in motion. The difference in frequency and its positive or negative character are elements that allow vectorial representation of blood speed and direction of movement against the transducer. Doppler ultrasound has several technical options: spectral and color coded, each of them with their advantages and disadvantages. Thus: spectral ultrasound describes the flow type (arterial or venous) and allows measurements. Color flow mode (CFM) ultrasound encodes speed vectors related to red blood cells groups thus detecting blood flows. Evaluation of tumor vasculature will be made first using the CFM module and then spectral Doppler module by positioning the Doppler sample on reference regions. On CFM investigation tumor vessels have tortuous paths, aberrant spatial ramifications, they intercommunicate and can abruptly end in "glove finger" (Cherrington et al, 2000). Spatial positioning of intratumoral vessels can also be illustrated by $3 \mathrm{D} / 4 \mathrm{D}$ reconstruction procedures and quantitative assessment can be achieved with techniques using color pixel count per unit area. Spectral investigation measurements into the tumor vessels lumen show either low values $(\mathrm{RI}<0.65 ; \mathrm{S} / \mathrm{D}<2.5)$ suggesting the absence of arterial precapillary sphincter and the presence of arterio-venous shunts or high values secondary to increased interstitial pressure. Accelerated flow speed can be identified in the input vessels from the tumor hilum suggesting a circulatory bed avid for blood (Cosgrove \& Eckersley, 1997). 


\subsubsection{Contrast Enhanced Ultrasound (CEUS)}

The introduction of intravenous (i.v.) contrast agents (CAs) is a great step forward for ultrasound. The first attempts to contrast ultrasound emerged in the '60s and had applications in interventional cardiology. After the 90s, first generation contrast agents were introduced into the clinical practice (the most used product is Levovist, Schering AG). These are air containing microbubbles, wrapped in a stabilizing membrane with the property to enhance the intensity of the echoes received from the blood flow thus making the Doppler signal more evident. This type of CAs has the disadvantage of a "blooming" effect of the color on CFM examination which masks the information derived from the microcirculation. With the development of transducer technology and the introduction of intermittent pulses emission with high mechanical index (MI> 0.2) it was possible to detect slow flows by identifying signals resulting from "breaking" microbubbles. This technique, called "destructive" contrast ultrasound is similar to that of contrast CT or MRI. Its disadvantage is that information cannot be obtained in "real time". After the year 2000, second generation contrast agents were introduced in clinical practice [SonoVue ${ }^{\circledR}$ in Europe, Sonazoid ${ }^{\mathrm{TM}}$ in Japan, Definity in Canada, Optison ${ }^{\mathrm{TM}}$ ). They consist of microbubbles filled with different gases other than air, with stability and elasticity in the bloodstream higher than that of the first generation CAs. The exposure of these CAs to low mechanical index (MI <0.1 to 0.2) US generates harmonics echoes resulting from non linear microbubble oscillation (table 1).

\begin{tabular}{|l|l|}
\hline \multicolumn{1}{|c|}{ Particularity } & \multicolumn{1}{c|}{ Importance } \\
\hline $\begin{array}{l}\text { They do not cross vessel walls, being } \\
\text { strictly confined to the circulatory } \\
\text { bed. }\end{array}$ & $\begin{array}{l}\text { They are real angiospecific tracers useful for } \\
\text { quantification of circulation in a region of } \\
\text { interest. }\end{array}$ \\
\hline $\begin{array}{l}\text { Characterized by repeated } \\
\text { recirculation from the large to small } \\
\text { circulation until complete clearance }\end{array}$ & High persistence and prolonged investigation \\
\hline $\begin{array}{l}\text { Elimination of contrast agent is made } \\
\text { through respiration and breakage }\end{array}$ & Can be used in renal and hepatic failure \\
\hline $\begin{array}{l}\text { They show a non-linear oscillation } \\
\text { when exposed to low MI ultrasounds }\end{array}$ & $\begin{array}{l}\text { Ultrasound information is found in harmonic } \\
\text { echoes } \\
\text { Ultrasound exploration is dynamic, "in real } \\
\text { time" }\end{array}$ \\
\hline
\end{tabular}

Table 1. Characteristics of second generation contrast agents. Particularities and importance.

As low acoustic power US are used, this procedure does not break the microbubbles, which allows "real time" blood flow examination during several minutes (Burns \& Wilson, 2006).

Harmonic contrast US examination, using second generation CAs requires special software equipment which suppresses 2D ultrasound image derived from the tissues by inverted phase ultrasound emission (the technique is called "pulse inversion"). In this way the information on the screen will correlate only to harmonic echoes generated by the microbubbles (Burns et al, 2000).

CEUS examination is a qualitative examination. As it is a continuous exploration, CEUS allows successive identification of vascular phases: arterial (approximately 10 - 15 sec. from 
the time of injection in the peripherals up to approx. 30-40 seconds) and venous. Some circulation areas (liver, spleen) have a "late" phase due to the "capture" of microbubbles into the reticulo-histiocyte system (RHS). In addition, the investigation also allows the assessment of vascular morphology which provides information similar to angiography. CEUS investigation has a high temporal resolution, superior to CT or MRI explorations.

CEUS liver investigation has two important elements that make it distinct (Cosgrove, 2007; Claudon et al, 2008):

a. the liver has a complex vascularization: for input (arterial and portal phase, the first can be identified in approx. 10 - 30 seconds after injection and the second in approx. 30 - 90 seconds after i.v. CA injection) and for elimination (sinusoidal phase, progressively occurs in approx. 120 seconds after injection and has a total duration of approx. 4-5 minutes);

b. liver tumors have a characteristic vasculature according to their nature, e.g. hepatocellular carcinoma is highly arterialized; metastases have a mixed input vasculature; benign tumors have a variable circulatory bed (hemangioma is highly capillarized). Tumor characterization using CEUS procedure is based on the contrast agent crossing the region of interest (dynamic, particularities) and on the vascular bed characteristics (presence, spatial distribution, vascular morphology, areas lacking signal which means scar or necrosis). This analysis is separately carried out in the arterial, portal venous and late phases. Algorithms are currently available that help liver mass discrimination (Bolondi et al, 2007). The procedure can be combined with 3D reconstruction techniques, which confers additional utility to the method (Luo et al, 2009).

A useful procedure to obtain more quantitative details is contrast curves analysis. These consist of graphical representation of the CA dynamics while crossing a region of interest. The method is useful for assessing chemotherapy efficacy in various malignancies.

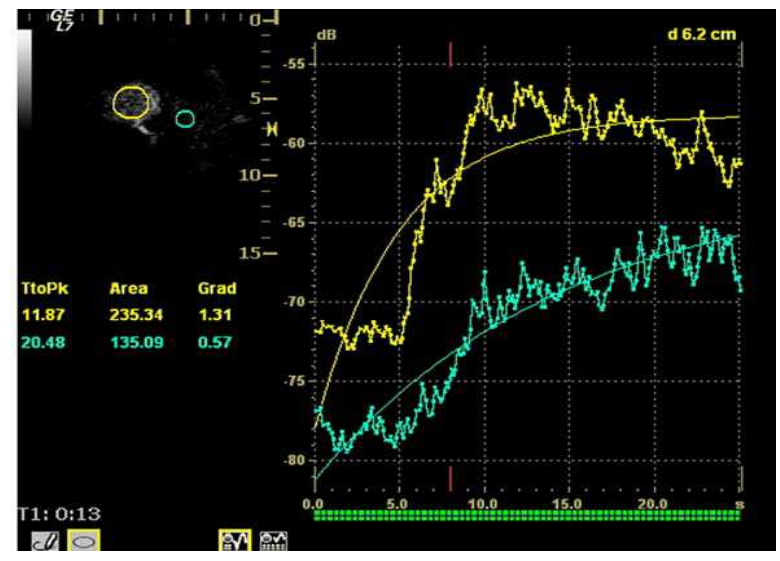

Fig. 1. Contrast dynamic analysis curves in the region of interest - TIC. Tracer dynamics in the region of reference is marked with blue and with yellow in the region of interest (tumor). It can be seen that the tumor has a more abundant circulation than the surrounding liver parenchyma. 
Limitations of CEUS examination. Harmonic contrast examination has some limitations including: a) the procedure is indicated only correlated with good 2D image; b) it has a relatively high cost; c) it is operator dependent examination.

\section{2D and harmonic ultrasonography in detecting and characterizing liver tumors}

\subsection{Characteristics of ultrasound diagnosis}

Ultrasound diagnosis of liver tumors involves two stages: detection and characterization.

Tumor detection is based on the performance of the method as already presented and should include morphometric information (three axes dimensions, volume) and topographic information (number, location specifying liver segment and lobe/lobes). The specification of these data is important for staging liver tumors and prognosis.

Tumor characterization is a complex process based on a sum of criteria leading towards tumor nature definition. Often, other diagnostic procedures, especially interventional ones are no longer necessary. Ultrasound examination has the same morphological and hemodynamic criteria as those of CT and MRI imaging procedures. However, semiology will be adjusted to the specifics of this method. Tumor characterization using the ultrasound method will be based on the following elements: consistency (solid, liquid, mixed), echogenity, structure appearance (homogeneous or heterogeneous), delineation from adjacent liver parenchyma (capsular, imprecise), elasticity, posterior acoustic enhancement effect, the relation with neighboring organs or structures (displacement, invasion), vasculature (presence, Doppler and CEUS characteristics). The substrate on which the tumor condition develops (if the liver is normal or if there is evidence of diffuse liver disease) and the developing context (oncology, septic) are also added. Particular attention should be paid to the analysis of the circulatory bed. Microcirculation investigation allows for discrimination between benign and malignant tumors. Characteristic elements of malignant circulation are vascular density, presence of vessels with irregular paths and size, some of them intercommunicating, some others blocked in the end with "glove finger" appearance, the presence of arterio-arterial and arterio-venous shunts, lack or incompetence of arterial precapillary sphincter made up of smooth musculatures (Weidener et al, 1991).

Diagnosis and characterization of liver tumors require a distinct approach for each group of conditions, using the available procedures discussed above for each of them. The correlation with the medical history, the patient's clinical and functional (biochemical and hematological) status are important elements that should also be considered.

\subsection{Benign liver tumors}

They generally develop on normal or fatty liver, are single or multiple (generally paucilocular), have distinct delineation, with increased echogenity (hemangiomas, benign focal nodular hyperplasia) or absent, with posterior acoustic enhancement effect (cysts), have distinct delineation (hydatid cyst), lack of vascularization or show a characteristic circulatory pattern, displace normal liver structures and even neighboring organs (in case of large sizes), are quite elastic and do not invade liver vessels. The patient has a good general status, as tumors are often asymptomatic, being incidentally discovered. 


\subsubsection{Liver cysts}

They can be single or multiple, with variable size, generally less than $20 \mathrm{~mm}$ (congenital). Rarely, sizes can reach several centimeters, leading up to the substitution of a whole liver lobe (acquired, parasitic). They may be associated with renal cysts; in this case the disease has a hereditary, autosomal dominant transmission (von Hippel Lindau disease). The ultrasound appearance is a well defined lesion, with very thin, almost unapparent walls, without circulatory signal at Doppler or CEUS investigation. The content is transonic suggesting fluid composition. The presence of membranes, abundant sediment or cysts inside is suggestive for parasitic, hydatid nature. Posterior from the lesion the "acoustic enhancement" phenomenon is seen, which strengthens the suspicion of fluid mass. They typically displace normal liver vessels but no vascular or biliary invasion occurs.
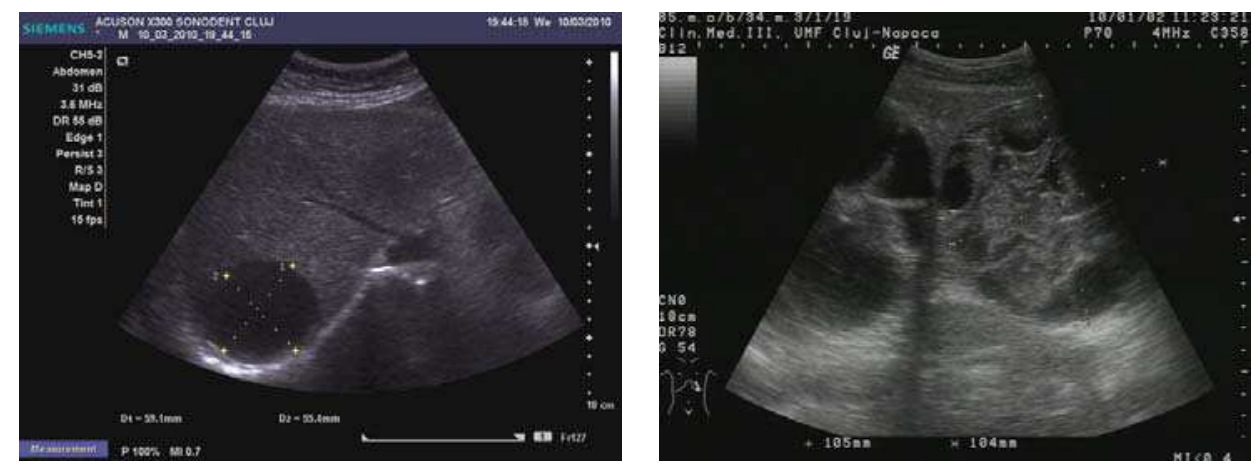

Fig. 2. Liver cyst - left image. Hydatid liver cyst (2D exam) - right image. Diagnostic criteria are the presence of membranes and sediment inside.

\subsubsection{Hemangioma}

It is the most common liver tumor with a prevalence of $0.4-7.4 \%$. It is generally asymptomatic but also can be associated with pain complaints or cytopenia and/or anemia when it is very bulky. It is unique or paucilocular. It can be associated with other types of benign liver tumors. Characteristic 2D ultrasound appearance is that of a very well defined lesion, with sizes of 2-3 cm or less, showing increased echogenity and, when located in contact with the diaphragm, a "mirror image" phenomenon can be seen. When palpating the liver with the transducer the hemangioma is compressible sending reverberations backwards. Doppler exploration reveals no circulatory signal due to very slow flow speed. CEUS investigation has real diagnosis value due to the typical behavior of progressive CA enhancement of the tumor from the periphery towards the center. The enhancement is slow, during several minutes, depending on the size of hemangioma and on the presence (or absence) of internal thrombosis. During late (sinusoidal) phase, if totally "filled" with CA, hemangioma appears isoechoic to the liver. Deviations from the above described behavior can occur in arterialized hemangiomas or those containing arterio-venous shunts. In these cases differentiation from a malignant tumor is difficult and requires other imaging procedures, follow up and measurements of the tumor at short time intervals. 


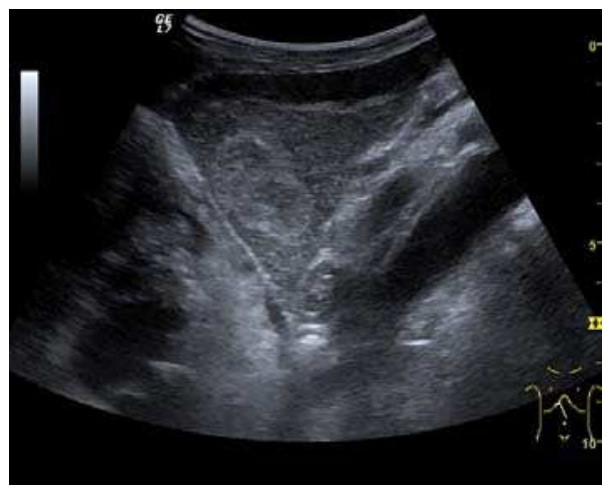

Fig. 3. Hepatic hemangioma (2D). The lesion is located in the left hepatic lobe. Note precise delineation, their increased echogenity and the heterogeneous internal structure.

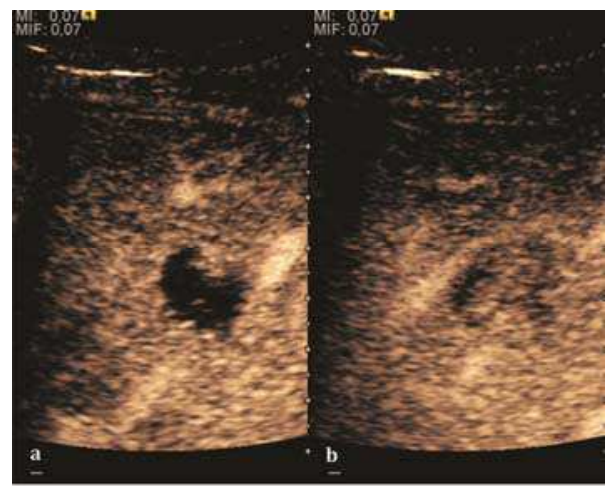

Fig. 4. Hepatic hemangioma (CEUS). Progression of CA from the periphery toward the center of the lesion is evidenced by examination at various time intervals ( $\mathrm{a}$ - arterial phase; $\mathrm{b}$ - late phase).

\subsubsection{Focal nodular hyperplasia}

It is a tumor developed secondary to a circulatory abnormality with abundant arterial vessels having a characteristic location in the center of the tumor, within a fibrotic scar. A radial vessels network develops from this level with peripheral orientation. The tumor's circulatory bed is rich in microcirculatory and portal venous elements. The incidence is higher in younger women and tumor development is accelerated by oral contraceptives intake. 2D ultrasound appearance is a fairly well-defined mass, with variable sizes, usually single, solid consistency with inhomogeneous structure. Rarely the central scar can be distinguished. Spectral Doppler examination detects central arterial vessels and CFM exploration reveals their radial position. CEUS examination shows central tumor filling of the circulatory bed during arterial phase and completely enhancement during portal venous phase. During this phase the center of the lesion becomes hypoechoic, enhancing the tumor scar. During the late phase the tumor remains isoechoic to the liver, which strengthens the diagnosis of benign lesion. 

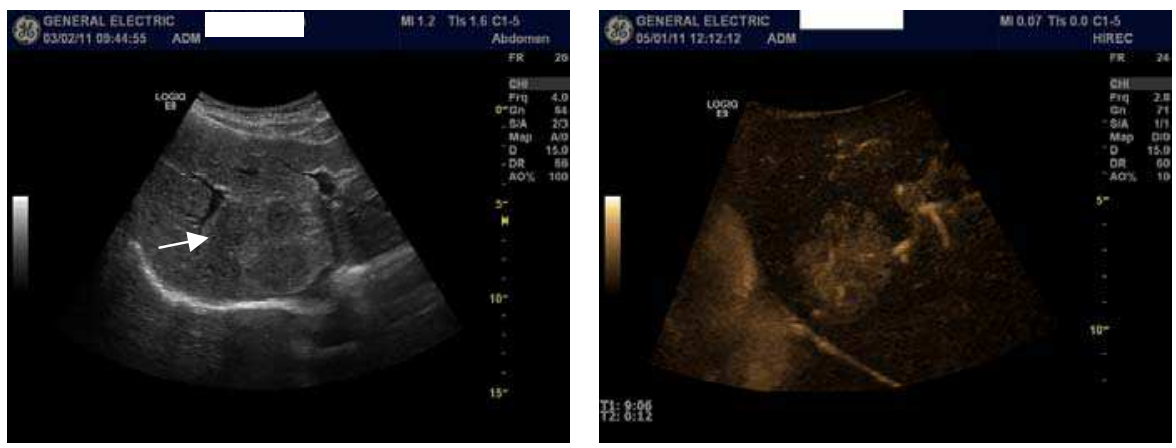

Fig. 5. Benign focal nodular hyperplasia (CEUS). Gray scale examination (left) detects the lesion. CEUS examination (right) allows characterization of tumor nature based on central contrast enhancement and centrifugal dispersion.

\subsubsection{Adenoma}

It is a benign tumor made up of normal or atypical hepatocytes. It has an incidence of $0.03 \%$. Its development is induced by intake of anabolic hormones and oral contraceptives. The tumor is asymptomatic, but may be associated with right upper quadrant pain in case of internal bleeding. 2D ultrasound shows a well-defined, un-encapsulated, solid mass. It may have a heterogeneous structure in case of intratumoral hemorrhage. Doppler examination shows no circulatory signal. CEUS exploration is quite ambiguous and cannot always establish a differential diagnosis with hepatocellular carcinoma. Thus, during the arterial phase there is a centripetal and inhomogeneous enhancement. During the portal venous phase there is a moderate wash out. During late phase the appearance is isoechoic or hypoechoic, due to lack of Kupffer cells.

\subsection{Malignant liver tumors}

Malignant liver tumors develop on cirrhotic liver (hepatocellular carcinoma, HCC) or normal liver (metastases). They are single or multiple (especially metastases), have a variable, generally imprecise delineation, may have a very pronounced circulatory signal (hepatocellular carcinoma and some types of metastases), have a heterogeneous structure (the result of intratumoral circulatory disorders, consequence of hemorrhage or necrosis) and are firm to touch, even rigid. The patient's general status correlates with the underlying disease (vascular and parenchymal decompensation for liver cirrhosis, weight loss, lack of appetite and anemia with cancer).

\subsubsection{Hepatocellular carcinoma (HCC)}

It is the most common liver malignancy (Parkin et al, 2005). It develops secondary to cirrhosis (in approx. 80\% of cases) (Llovet et al, 2003) therefore, ultrasound examination every 6 months combined with alpha fetoprotein (AFP) determination is an effective method for early detection and treatment monitoring for this type of tumor (Bruix \& Sherman 2005; Llovet \& Bruix 2008). Clinically, HCC overlaps with advanced liver cirrhosis (long evolution, repeated vascular and parenchymal decompensation, sometimes bleeding 
due to variceal leakage) in addition to accelerated weight loss in the recent past and lack of appetite.

HCC appearance on 2D ultrasound is that of a solid tumor, with imprecise delineation, with heterogeneous structure, uni- or multilocular (encephaloid form). An "infiltrative" type is also described which is difficult to discriminate from liver nodular reconstruction in cirrhosis. Typically HCC invades liver vessels, primarily the portal veins but also the hepatic veins (Badea R. \& Badea Gh, 1991). Doppler examination detects a high speed arterial flow and low impedance index (correlated with described changes in tumor angiogenesis). The spatial distribution of the vessels is irregular, disordered. CEUS examination shows hyperenhancement of the lesion during the arterial phase. During the portal venous phase there is a specific "wash out" of ultrasound contrast agent (UCA) and the tumor appears hypoechoic during the late phase. Poorly differentiated tumors may have a stronger wash out leading to an isoechoic appearance to the liver parenchyma during portal venous phase. This appearance was found in approx. $30 \%$ of cases (Nicolau et al, 2004). The described changes have diagnostic value in liver nodules larger than $2 \mathrm{~cm}$.

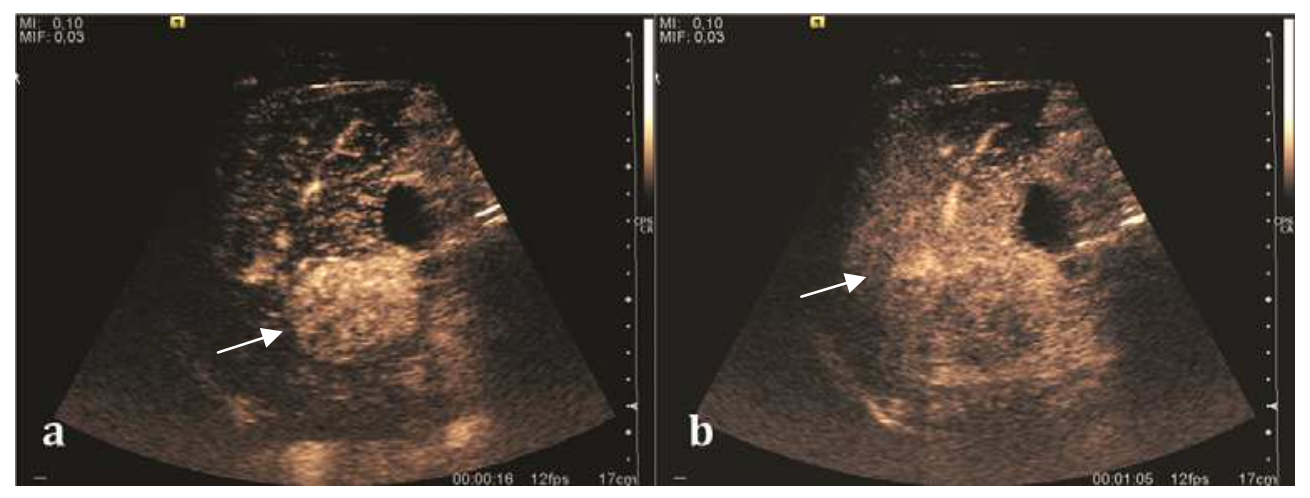

Fig. 6. Encephaloid hepatocellular carcinoma (CEUS). Contrast tumor enhancement is observed on the left during arterial phase. The "wash-out" phenomenon can be seen on the right, during portal venous phase.

Ultrasound is useful in HCC detection, stadialization and assessing therapeutic efficacy. In terms of staging related to therapy effectiveness, the Barcelona classification is used (Llovet et al, 1999) which identifies five HCC stages. Curative therapy is indicated in early stages, which include very early stage (single nodule $<2 \mathrm{~cm}$ ), curable by surgical resection (survival 50-70\% five years after surgical resection) (Llovet et al, 2003) and early stage (single nodule of $2-5 \mathrm{~cm}$, or up to 3 nodules $<3 \mathrm{~cm}$ ) which can be treated by radiofrequency ablation (RFA) and liver transplantation. Intermediate stage (polinodular, without portal invasion) and advanced stage (N1, M1, with portal invasion) undergo palliative therapies (TACE and sorafenib systemic therapy) and in the end stage only symptomatic therapy applies. 


\subsubsection{Cholangiocarcinoma}

It develops on non cirrhotic liver. 2D ultrasound appearance is uncharacteristic - solid mass with heterogeneous structure, poorly delineated, often with peripheral location and weak Doppler circulation signal. CEUS examination reveals a moderate enhancement of the tumor periphery during arterial phase followed by wash-out during portal venous phase and hypoechoic appearance during late phase.

\subsubsection{Liver metastases}

US examination is required to detect liver metastases in patients with oncologic history. In addition, the method can incidentally detect metastases in asymptomatic patients. Early identification (small sizes, small number) is important to establish an optimal course of treatment which can be complex (chemotherapy, radiofrequency ablation, surgical resection) but welcomed. In addition, discrimination of synchronous lesions that have a different nature is also important knowing that up to $25-50 \%$ of liver lesions less than $2 \mathrm{~cm}$ detected in cancer patients may be benign (Kreft et al, 2001). US sensitivity for metastases detection varies depending on the examiner's experience and the equipment used and ranges between $40-80 \%$ (Wernecke et al, 1991). Sensitivity is conditioned by the size and acoustic impedance of the nodules. For a lesion diameter below $10 \mathrm{~mm}$ US accuracy is greatly reduced, reaching approx. 20\%. Other elements contributing to lower US performance are: excessive obesity, fatty liver disease, hypomobility of the diaphragm, and certain patterns of hyperechoic or isoechoic metastases that can be overlooked or can mimic benign conditions. Conventional US appearance of metastases is uncharacteristic, consisting of circumscribed lesions, with clear, imprecise or "halo" delineation, with homogeneous or heterogeneous echo pattern. They can be single (often liver metastases from colonic neoplasm) or multiple. Echogenity is variable. When increased, they can compress the bile ducts (which may be dilated) and the liver vessels. Liver involvement can be segmental, lobar or generalized. In this situation a pronounced hepatomegaly occurs. Generally, metastases have non-characteristic Doppler vascular pattern, with few exceptions (carcinoid metastases). Cyst-adenocarcinoma metastases due to semifluid content may have a transonic appearance. When increasing, they can result in central necrosis. CEUS examination is a real breakthrough for detection and characterization of liver metastases. Increased performance is based on identifying specific vascular patterns during the arterial phase and seeing metastases in contrast to normal liver parenchyma during the sinusoidal phase. CEUS increased accuracy is due to the different behavior of normal liver parenchyma (captures CA in Kuppfer cells) against tumor parenchyma (does not contain Kuppfer cells, therefore CEUS appearance is hypoechoic). To this adds the particularities of intratumoral circulation represented by a reduced arterial bed compared to that of the surrounding normal liver and the absence of the portal vessels (Cosgrove \& Blomley 2004). In terms of vascularity, metastases can be hypovascular (in gastric, colonic, pancreatic or ovarian adenocarcinomas) with hypoechoic pattern during arterial phase, and similar during portal venous and late phases, respectively hypervascular (neuroendocrine tumors, malignant melanoma, sarcomas, renal, breast or thyroid tumors) with hyperechoic appearance during arterial phase, with washout during the portal venous phase and hypoechoic pattern 30 seconds after injection (Larsen, 2010). 

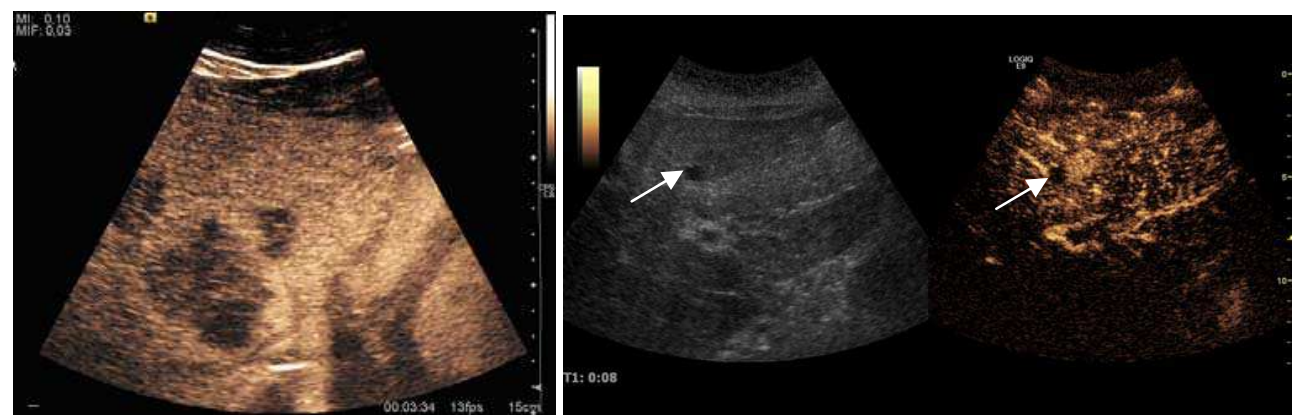

Fig. 7. Liver metastases (CEUS). Peripheral vascular pattern of the lesion is observed on the left in colon cancer metastasis. Lesion hyperenhancement in ovarian cancer liver metastasis is seen on the right during the arterial phase.

Using CEUS examination to detect metastases a sensitivity of $80-95 \%$ is obtained, similar to that of contrast CT and MRI (Quaia et al, 2006 ; Piscaglia et al, 2007). Intraoperative use of the procedure increases its performance even if it does not have a decisive contribution to change the therapeutic behavior (Konopke et al, 2005). Limitations of the method are those related to US penetration (pronounced fatty liver disease, deep lesion, excessive obesity) and to the experience of the examiner. To this the risk of confusion between hypervascular metastases, hepatocellular carcinoma and hemangioma and the confusion between hypovascular metastases and small liver cysts is added. Routine use of CEUS examination to detect liver metastases is recommended when conventional US examination is not conclusive, when precise information on some injuries (number, location) is necessary in conjunction with contrast CT/MRI and to assess the effectiveness of treatment when using a antiangiogenic therapy for hypervascular metastases (Claudon et al, 2008). The method cannot replace CT/MRI examinations which have well established indications in oncology (Larsen, 2010).

\subsection{Pseudotumors and inflammatory masses of the liver}

Besides the entities listed above inflammatory masses or even pseudo-masses can occur. Their diagnosis is quite difficult and the criteria used for differentiation are often insufficient, requiring morphologic diagnostic procedures, use of other diagnostic imaging methods or patient reevaluation from time to time. This includes lesions developed on liver parenchyma reconstruction, as occurs in cirrhosis, steatosis accumulation or in case of acute or chronic inflammatory diseases.

\subsubsection{Focal steatosis}

It consists of localized accumulation of fat-rich liver cells. In some cases this accumulation can mimic a liver tumor. Sometimes the opposite phenomenon can be seen, that is an "island" of normal parenchyma in a "shining" liver. In both cases ultrasound examination identifies a well defined, un-encapsulated area, with echostructure and vasculature similar to those of normal liver parenchyma. The lesion can have different forms, most cases being oval and located in the IVth segment, anterior from the hepatic hilum. It occurs in dyslipidemic or 
alcohol intake patients with normal physical and biological status. Benign diagnosis confirmation is made using CEUS examination which proves a normal circulatory bed similar to adjacent liver parenchyma in all three phases of investigation (Molins et al, 2010).

\subsubsection{Liver abscess}

Liver abscess have heteromorphic ultrasound appearance, the most typical being that of a mass with irregular shapes, fringed, with fluid or semifluid content, with or without air inside. Doppler examination shows the lack of vessels within the lesion. CEUS exploration shows hyperenhancement during arterial phase close to the lesion, this being suggestive of a liver parenchymal hyperemia. During venous and sinusoidal phase the pattern is hypoechoic, and the central fluid is contrast enhanced. CEUS examination is useful because it confirms the clinical suspicion of abscess. In addition, it allows for an accurate measurement of the collection size and an indication regarding its topography inside the liver (lobe, segment).

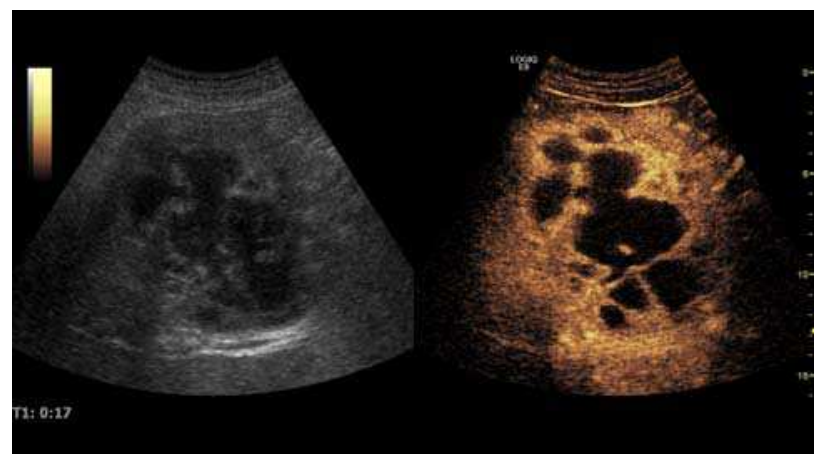

Fig. 8. Liver abscess (2D and CEUS). 2D Examination reveals the fluid nature of the mass and imprecise delineation. CEUS examination shows congestion in the surrounding liver parenchyma and excludes a vascular tumor.

\section{Preneoplastic status. Cirrhotic liver monitoring}

Cirrhotic liver is characterized by the occurrence of nodules with different sizes and evolution degrees, so that regenerative nodules, dysplastic nodules and even early hepatocellular carcinoma can coexist at some moment during disease progression. There are studies showing that between $59-94 \%$ of newly diagnosed liver nodules in cirrhotic patients have malignant histology and up to $50 \%$ of hyperechoic lesions, with ultrasound appearance of hemangioma, ultimately prove to be hepatocellular carcinoma. Therefore, current practice in many centers considers that any new lesion revealed in a cirrhotic patient should be regarded as malignant until otherwise proven (Andreana et al, 2009). There are three categories of cirrhotic liver nodules: regenerative, dysplastic (considered as premalignant conditions) and tumoral (HCC) (Int WP, 1995).

\subsection{Regenerative nodules (RN)}

These lesions are well defined, with isoechoic or hypoechoic appearance and sizes less than $1 \mathrm{~cm}$. They are high in numbers and have a more or less uniform distribution, involving all 
liver segments. They can crowd resulting in large pseudo tumors. At Doppler examination, these nodules have no circulatory signal. CEUS exploration is indicated when a nodule is different against the general pattern of restructured liver either by different echogenity or by a different size than the majority of nodules. During the arterial phase, the signal is weak or absent. During the portal venous and late phase, the appearance is persistently isoechoic. Generally, RN is not distinct from the surrounding parenchyma. CEUS examination is useful to exclude an active lesion at the moment of exploration but does not have absolute prognostic value; therefore the patient should be periodically examined at short intervals (Kojiro, 2004; Bolondi et al, 2005). Correlation with clinical status and AFP measurements is required.

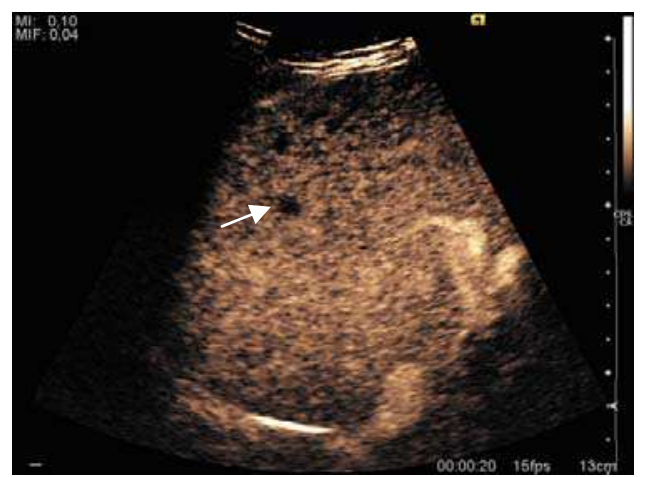

Fig. 9. Regenerative nodule (CEUS). One can see the hypovascular pattern of the solid nodule, with a size $<10 \mathrm{~mm}$.

\subsection{Dysplastic nodules (DN)}

These lesions have various patterns (hypo or hyperechoic) with at least $1 \mathrm{~cm}$ diameter. They are hepatocytes with dysplastic changes, but without clear histological criteria for malignancy. They are divided into low-grade dysplastic nodules, where cellular atypia are mild and high-grade dysplastic nodules with moderate or severe cellular atypia, but without any established signs of malignancy. Occasionally, well-differentiated HCC foci can be identified in high-grade dysplastic nodules (appearance called "nodule in nodule") (Minami \& Kudo, 2010). Most authors accept the carcinogenesis process as a progressive transformation of DN from low-grade to high-grade and into HCC. The nodule's vasculature changes progressively, correlated with the degree of malignancy, and it is characterized by decrease until absence of portal venous input and by increase of arterial intratumoral input. Neoformation vessels occur with increasing degree of dysplasia. Arterial neovascularization is enhanced in a chaotic and explosive way, while normal, arterial and portal vasculature continues to decline. High-grade dysplastic nodules are hypovascularized both arterial and portal phases, while early HCC nodules may have similar arterial pattern with the surrounding parenchyma or exacerbated, and portal hypovascularization. In moderate or poorly differentiated HCC (classic HCC) tumor nutrition is performed only by neoformation vessels (abundant), the normal arterial and portal vasculature completely disappearing (Matsui 2004). This behavior of intratumoral vascularization is typical for HCC and is the key to imaging diagnosis (Lencioni et al 2008). 
B-mode ultrasonography is unable to distinguish between regenerative nodules and "borderline" lesions such as dysplastic nodules and even early HCC. Doppler examination also has a low sensitivity in differentiating dysplastic nodules from early HCC. Doppler signal may be absent in both regenerative and dysplastic nodules. Some authors indicate the presence of venous type Doppler flow which reflects the portal venous nutrition of the nodule as a characteristic feature of dysplastic nodules and early HCC (Minami \& Kudo, 2010). Other authors noticed the presence of an arterial flow with small frequency variations and a normal resistivity index (RI) (Lencioni et al 2008).

On CEUS examination both RN and DN may have quite a variable enhancement pattern. Generally, both nodules enhances identically with the surrounding liver parenchyma after UCAs injection. Dysplastic nodules are hypovascular in the arterial phase. In case of highgrade dysplastic nodule sometimes a hypervascularization can be detected, but without associating "wash out" during portal and late CEUS phases. In these cases, biopsy may clarify the diagnosis.
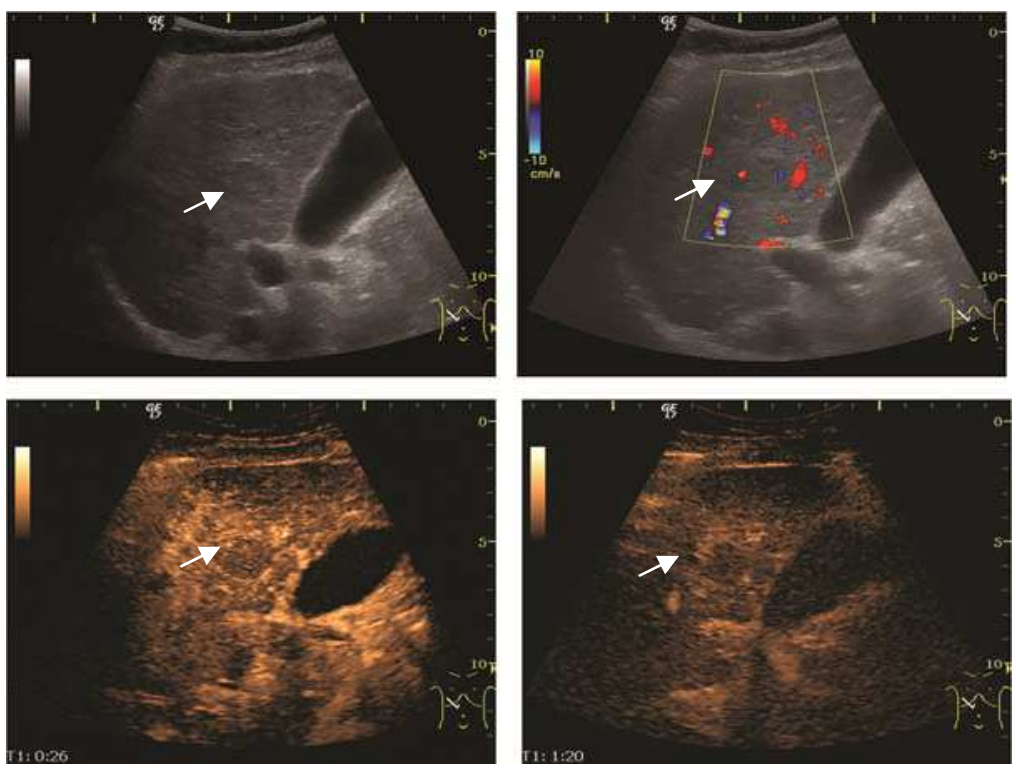

Fig. 10. Dysplastic nodule (2D, CFM, CEUS). The nodule is well-defined with a size between 10- $20 \mathrm{~mm}$, lacks vessels in CFM and CEUS exploration.

\subsection{Early hepatocellular carcinoma (Early HCC)}

The suggestive appearance of early HCC on 2D ultrasound examination is that of hypoechoic nodule, with distinct pattern, developed on cirrhotic liver. Hypoechoic appearance is characteristic of moderate/poorly differentiated HCC, with low or absent fatty changes. Rarely, HCC may appear isoechoic, consist of a tumor type with a higher degree of differentiation and therefore with slower development. Another common aspect is "bright loop" or "nodule-in-nodule" appearance, hypoechoic nodules in a hyperechoic tumor. (Minami \& Kudo, 2010). 
Spectral Doppler characteristics of early HCC overlap those of the dysplastic nodule, as they are represented by the presence of portal venous signal type or arterial type with normal RI (well differentiated HCC) or increased RI (moderately or poorly differentiated HCC). The CFM exploration identifies a chaotic vessels pattern.

On CEUS examination, early HCC has an iso- or hypervascular appearance during the arterial phase followed by wash out during portal venous and late phase. There are studies showing that the wash out process is directly correlated with the size and features of neoplastic circulatory bed. Thus, highly differentiated HCC illustrates the phenomenon of late or even very late "wash out" while poorly differentiated HCC has an accelerated wash out at the end of arterial phase (Strobel et al, 2005; von Herbay et al, 2009; Jang et al, 2009). It is therefore mandatory to analyze all these three phases of CEUS examination for a proper characterization of liver nodules. Tumor wash out at the end of the arterial phase allows the HCC diagnosis with a predictability of $89.5 \%$. Some authors consider that early pronounced contrast enhancement of a nodule within 1-2 cm developed on a cirrhotic liver is sufficient for HCC diagnosis (Jang et al, 2009). These results prove that for a correct characterization of the lesions it is necessary to extend the examination time to 5 minutes or even longer (von Herbay et al, 2009).

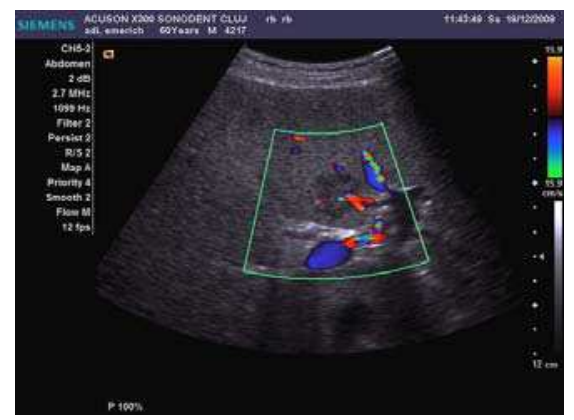

Fig. 11. Early hepatocellular carcinoma (2D, CFM). The 2D examination reveals a solid, hypoechoic nodule in IVth liver segment, without encapsulation. CFM shows a central vessel with ramifications to the periphery. The underlying liver is cirrhotic.

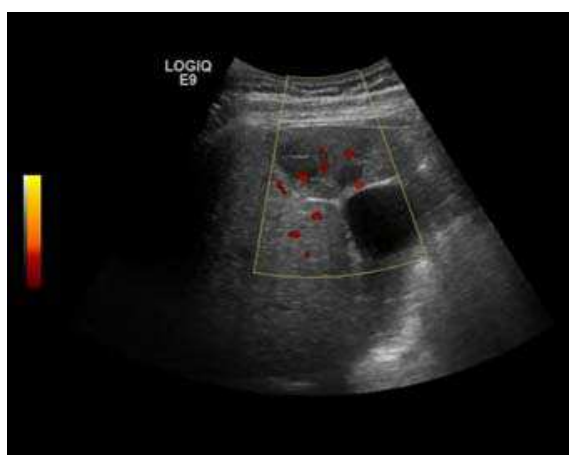

Fig. 12. Early hepatocellular carcinoma (2D, CFM). “Nodule in nodule” image: small hypoechoic early HCC inside monitored dysplastic nodule. 


\subsection{The ultrasound value in HCC "screening"}

Baseline 2D ultrasound has an important role in surveillance programs for patients at risk to develop HCC (Bruix \& Sherman, 2011). The examination has an acceptable sensitivity which increases with the tumor size. Sensitivity varies between $42 \%$ for lesions $<1 \mathrm{~cm}$ and $95 \%$ for tumors larger than $1 \mathrm{~cm}$, and specificity can reach 90\% (Andreana et al, 2009). Optimal time interval for ultrasound screening of "at risk" population is 6 months as it results from clinical trials that investigated the tumor size doubling time (Bruix, 2005; Maruyama et al, 2008). For a recently developed nodule the dimensional criteria will be taken into account. Thus, for a nodule with a size of less than $10 \mathrm{~mm}$ the patient will be reevaluated by ultrasound every 3 months, as the growth trend is an indication for completion of investigations with other diagnostic procedures; at a size between $10-20 \mathrm{~mm}$ two concordant imaging procedures are necessary, supplemented if necessary by an ultrasound guided biopsy; at a size over $20 \mathrm{~mm}$ one single dynamic imaging technique with characteristic appearance is enough for positive diagnostic. In uncertain cases complementary dynamic imaging techniques or biopsy should be performed. When Doppler exploration is not enough, CEUS examination will be performed (Gaiani et al, 2001). One should always keep in mind the risk of false positive results for HCC in case of cholangiocarcinomas so complementary diagnostic procedures should be considered (Bruix \& Sherman, 2011).

The effectiveness of screening programs is proved by an increase in detection rate of HCC $<2 \mathrm{~cm}$ (from $<5 \%$ in the $90 \mathrm{~s}$ in Europe to $>30 \%$ today in Japan) with curative therapy options (Llovet \& Bruix 2008). The main problem of ultrasound screening is that, in order to be cost-effective, it should be applied to the general population and not in tertiary hospitals. This raises the importance of the operator and equipment dependent part of the ultrasound examination (Bruix et al, 2001). The efficiency of such a program is linked to the functional liver parenchyma of the cirrhotic patient. Therefore, some authors argue that screening should be excluded in patients with etiologies that prevent curative treatment or in patients with advanced liver disease (Child-Pugh class C) (Zapata et al, 2010).

After curative therapies (surgical resection, local ablative therapies) continuing ultrasound screening is recommended first at 1 month then at 3 months intervals after the therapy to assess the effectiveness of therapy and to detect other nodules.

\section{Antitumor therapies}

Ultrasound exploration can be an effective procedure for the assessment of liver tumors response to treatment. Over the years, different criteria for assessing the effectiveness of curative or palliative therapies have been considered. Now it has been proved that the degree of tumor necrosis is not correlated with tumor diameter, therefore simple measurement of the tumor diameter (RECIST criteria) is not enough for therapy assessment. Currently, local response to treatment is focused on tumor necrosis diagnosed by contrast dynamic imaging techniques and recognized by the presence of intratumoral non-enhanced areas. Local response to treatment is defined as:

a. complete response, defined as complete disappearance of all known lesions (absence of tumor enhanced areas, reflecting total tumor necrosis) and absence of other new lesions determined by two observations not less than 4 weeks apart; 
b. partial response, defined as more than $50 \%$ reduction in total tumor enhancement in all measurable lesions, determined by two observations not less than 4 weeks apart

c. stable disease (is not described by $\mathrm{a}, \mathrm{b}$, or $\mathrm{d}$ )

d. progressive disease, defined as $25 \%$ increase in size of one or more measurable lesions or the appearance of new lesions (Bruix et al, 2001).

\subsection{Techniques for evaluating the efficiency of therapy}

The efficiency of 2D ultrasound is low in assessing the effects of HCC or metastasis therapy, as it is unable to differentiate viable tumor tissue from post-therapy tumor necrosis. However, it is able to detect the appearance of new lesions and to assess the occurrence of any complications of disease progression (ascites or portal vein thrombosis). Color Doppler ultrasound can be useful sometimes being able to show the presence of intratumoral vasculature as a sign of incomplete therapy or intratumoral recurrence. The absence of Doppler signal does not exclude the presence of viable tumor tissue. CEUS exploration, by its ability to enhance intra-lesion microcirculation, has proved its utility in monitoring therapeutic efficacy. Its indications are defined for HCC ablative treatments (pre, intra and post-therapy), while monitoring of systemic therapies of HCC and metastases are not validated indications at this time, but with proved efficacy in extensive clinical trials (Claudon et al, 2008). CEUS examination cannot completely replace the other imaging diagnostic methods currently in use because of the known limitations of the ultrasound method (operator/ equipment dependent, ultrasound examination limitations). In addition to bloating, in cancer patients post-therapy steatosis occurs, which prevent deep visibility. Spiral CT scan remains the method of choice in monitoring cancer therapies because it provides an overview of tumor extension and it is not limited by bloating or steatosis (Bartolozzi et al, 1999).

Gadolinium MRI examination is a procedure used more and more often, and its advantages are the absence of irradiation and its high sensitivity in tumor vasculature detection, especially in smaller tumors (Dromain et al, 2002). However it remains an expensive and not a very accessible procedure, although it has a high specificity. Currently, CEUS and MRI are considered complementary methods to CT scan.

\subsection{Ultrasound monitoring ablative therapies (alcoholization - PEI, radiofrequency ablation - RFA)}

Ablative therapies are considered curative treatments for HCC together with surgical resection and liver transplantation and they are indicated for early tumor stages in patients with good liver function (Bruix \& Sherman, 2005; Bruix \& Sherman, 2011). Also they are successfully applied in the treatment of liver metastases, where surgical resection is contraindicated. They are chemical (intratumoral ethanol injection) or thermal (radiofrequency, laser or microwave ablation). They are applied in order to obtain a full therapeutic response, without affecting liver function. Complete response is locally proved by complete tumor necrosis with a safety margin around the tumor.

2D ultrasound, Doppler ultrasound and especially CEUS can play an important role in pretherapeutic staging, particularly when sectional imaging investigations (CT, MRI) provide uncertain results or are contraindicated. During the interventional procedure, ultrasound 
allows guidance of the needle into the tumor. CEUS allows guidance in areas of viable tissue and avoids intratumoral necrotic areas. CEUS also allows assessment of therapeutic effect immediately post-procedure (with the possibility of reintervention in case of partial response) (Claudon et al, 2008). To accurately assess the effectiveness of treatment it is mandatory to compare the tumor diameter before therapy with the ablation area. The volume of damaged tissue must be higher than the initial tumor volume. CEUS appearance is that of central nonenhanced area showing a peripheral homogeneous hyperenhanced rim due to post-procedure inflammation. 24 hours after the procedure the inflammatory peripheral rim is thinning and the necrotic area appears larger than at the previous examination. Thus, a possible residual tumor may appear more evident. Residual tumor has poorly defined edges, irregular shape, and the tumor diameter is unchanged. Residual tumor tissue is evidenced at the periphery of the tumor as an eccentric area behaving as the original tumor at CEUS examination, with arterial hyperenhancement and portal and late wash-out. Ultrasound examination 24 hours after the procedure, including CEUS, can show apart from the character of the lesion any potential post-intervention complications (e.g. active bleeding).

In the first days after RFA both CEUS and spiral CT have low sensitivity in assessing therapeutic efficacy. CT sensitivity 24 hours post-therapy is reported to be even lower than CEUS (Vilana et al, 2006). Difficulties in CEUS examination result from post-lesion hyperemia, presence of intratumoral air, ultrasound limitations (too deep lesion or the presence of fatty liver) or lack of patient's cooperation (immediately after therapy). For this reasons contrast imaging (CT or CEUS) control should be performed one month after ablation to confirm the result of the therapy (Spârchez et al, 2009).

Local recurrence is defined as recurrence of a hyperenhanced area at tumor periphery in the arterial phase, with portal and late wash-out. Sometimes, especially for HCC treated by alcoholization (PEI) hyperenhanced septa or vessels can be shown inside the lesion (Spârchez et al, 2009).

In case of successful treatment, US monitoring using CEUS is performed every three months. Although CE-CT and/or MRI are considered the method of choice in post-therapy monitoring, CEUS can be used in follow-up protocols (Claudon et al, 2008), its diagnostic accuracy being equivalent to that of CE-CT or MRI (Frieser et al, 2011).
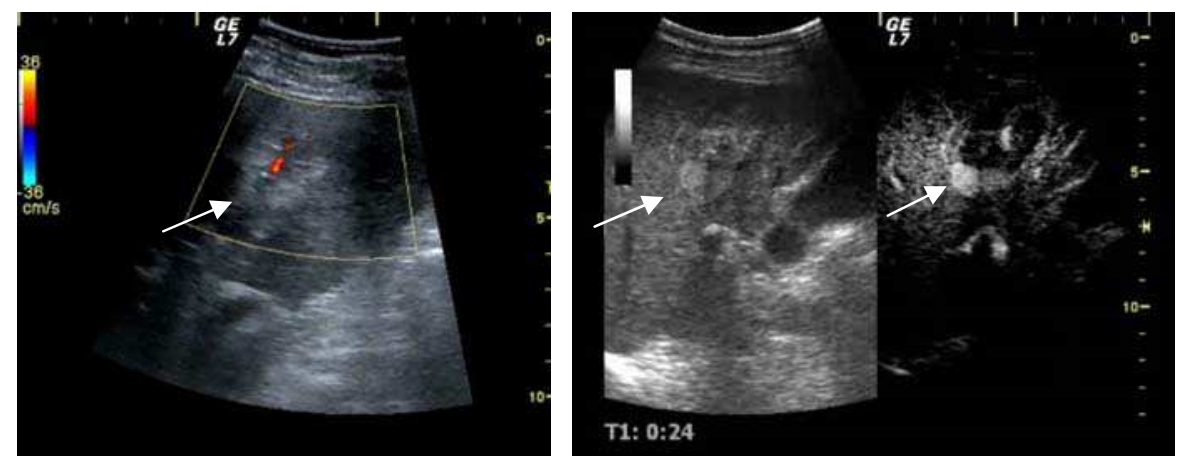

Fig. 13. Assessment of therapeutic efficacy on ultrasound (2D, CFM, CEUS). US exam shows vascular Doppler signal at CFM (left) and CEUS examination reveals incomplete therapy (right). 


\subsection{Ultrasound monitoring of TACE therapy (transarterial chemoembolization)}

Transarterial chemoembolization (TACE) is part of palliative therapies for HCC used in intermediate stages of the disease. It consists of selective angiographic catheterization of the hepatic artery and injection of chemotherapeutic agents (usually adriamycin, but other molecules are currently the subject of clinical trials), followed by embolization of hepatic artery with gelfoam, alcohol or metal rings (Bruix \& Sherman, 2005). A similar procedure is transarterial embolization but without chemotherapeutic agents injection, used in the treatment of hypervascular liver metastases. These therapies are based on the predominantly arterial vasculature of HCC and hypervascular metastases, while the remaining liver parenchyma has a dual vascular intake, predominantly portal. Their efficacy is high only for lesions who are hyperenhanced during arterial phase. The role of US is limited in the first few days after the procedure, and refers only to its complications, due to Lipiodol retention mainly intratumoral, but also diffusely intrahepatic. On ultrasound, Lipiodol appears intensely hyperechoic inside the tumor, with significant posterior attenuation which make US examination more difficult. On the other hand, CE-CT is also limited by the presence of Lipiodol (iodine oil), therefore the evaluation of therapeutic efficiency is currently made by indirect assessing Lipiodol binding to the tumor using nonenhanced CT (Maruyama et al, 2008). CE-MRI is not influenced by the presence of Lipiodol, but it is an expensive method and still difficult to reach. Several studies have proved similar efficacy, even superior, of CEUS compared to CE-CT and CE-MRI for the evaluation of postTACE treatment results, while other studies have shown the limitations of CEUS especially for deep or small lesions. Given the CEUS limitations, currently some authors consider CT as standard method for the evaluation of TACE and local ablative therapies and CEUS and CE-MRI as complementary methods (Lim et al, 2006, Maruyama et al, 2008). Monitoring TACE therapeutic results by contrast imaging techniques is performed as for ablative therapies initially after one month then after every 3 months post-TACE.

Given that TACE is indicated only for hyperenhanced lesions during arterial phase, CEUS plays a very important role in monitoring the dysplastic nodules to identify the moment when changes occur in arterial vasculature, being able to have an early therapeutic intervention in order to limit tumor progression, to increase patient survival, and thus to create a bridge to liver transplantation.
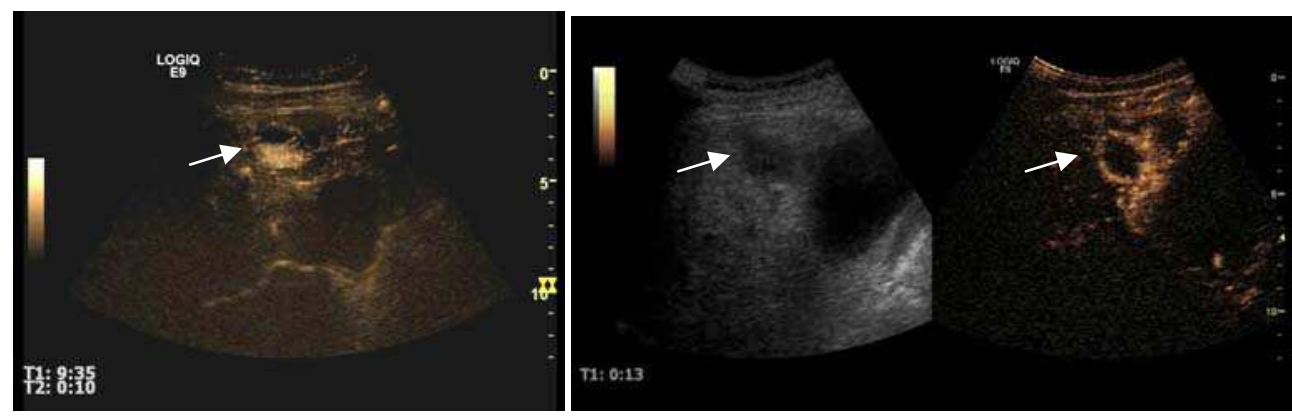

Fig. 14. Small HCC is seen on the left (nodule in nodule). Efficient chemoembolization of the nodule (right). CEUS examination shows no circulatory signal within the nodule. 


\subsection{Ultrasound monitoring of systemic therapies}

Systemic therapies are procedures based on the affinity of certain molecules to inhibit either tumor cell replication or multiplication of neoplastic vasculature (antiangiogenic therapies). They are intravenously administered and are indicated in advanced stages of liver tumor diseases, when there are no other effective therapeutic solutions. Among ultrasound techniques, CEUS is the one that brought a significant benefit not only by increasing the sensitivity and specificity of ultrasound in detecting liver metastases, but also by assessing the efficacy of systemic therapy for HCC and metastases. The method has been adopted by oncologists since 2003, because it involves no irradiation and has no hepatic or renal toxicity, and it is now currently used in tumor therapeutic evaluation (Lassau et al, 2011). It is currently used in large clinical trials aimed at determining the efficacy of different types of anti-angiogenic molecules by quantifying intratumoral perfusion based on the statistical analysis performed using specific software during post-processing in order to assess therapeutic efficacy as early as possible.

\subsection{Ultrasound monitoring of post-surgical resection status}

Surgical resection is the treatment of choice for non-cirrhotic or cirrhotic patients with well preserved liver function with single liver lesion. After resection, recurrence rate exceeds $70 \%$ at 5 years and is mainly due to primary tumor dissemination. The strongest predictors of tumor recurrence are the development of other tumors near the primary tumor scar and vascular microinvasion (Bruix \& Sherman, 2005). 2D ultrasound is used within screening programs. CEUS efficacy has not been proven so far in monitoring postresection patients.

\section{Other applications of ultrasound in liver tumor pathology. Technical progress}

Ultrasound has known a great development arising from the need to increase patient access to advanced investigations while avoiding procedures using radiation (CT), contrast agents with allergic potential (iodine substances) and hepatic or renal toxic agents.

Interventional and intraoperative ultrasound. Ultrasound exploration by maneuverability of the equipment and dynamic character of the image allows guided interventional procedures or intraoperative examination. Combining ultrasound with such procedures is necessary due to the lack of specificity of the method in case of tumors. Even if tumor markers for defining the nature of some masses are available, their accuracy is not good enough; therefore biological samples from the nodules detected by imaging is often required. Accuracy of ultrasound guided puncture in the diagnosis of liver tumors is dependent on the operator's experience and can reach up to 87.5 - 99\% (Horigome et al, 1999). The extracted material can be either a histology fragment or cytology aspiration; in both cases the credibility of the method is sufficient to ensure optimal cancer therapies (Badea R \& Badea Gh 1991). Intraoperative ultrasound is also a complementary investigation that allows detection of very small nodules. In addition it allows the characterization of tumor using Doppler and CEUS procedure as well as guided interventional procedures such as intraoperative PEI or RFA. 
Targeted therapy using contrast agents. The introduction of second generation contrast agents has been a great progress. Diagnostic performance is already proven. A step forward is tumor targeted therapy under ultrasound guidance. The principle is that of transporting chemotherapeutic agents to the target using microbubbles as vectors. Experiments have been made for binding different substances and/or genes to the lesion, with local release by "breaking" and intracellular penetration using the phenomenon of cellular membrane permeability called "sonoporation" (Lindner, 2004, Newman \& Bettinger, 2007).

Image fusion. Techniques for image fusion obtained by different imaging procedures allow the correlation of real-time ultrasound examination with CT or MRI images, enabling positioning of the needle in relation to the exact position of the tumor. The technique allows a better guidance both for biopsies and percutaneous ablative procedures, replacing CT guidance and thus avoiding irradiation. Combination with CEUS allows a better characterization of lesions as well as successful monitoring of percutaneous procedures or TACE effects (Sandulescu et al, 2011; Ewertsen et al, 2011).

Elastography. It was initially introduced in practice to assess the degree of fibrosis in chronic liver disease. In liver tumors it allows detection of liver nodules due to the difference of elasticity between the hepatic nodule and the parenchyma. There are few studies that try to assess the benign or malignant character of the lesion based only on elasticity. By combination with CEUS the method could be beneficial for early detection and characterization of HCC.

High Intensity Focused Ultrasound (HIFU). It is a new technique capable of destroying tumor tissue by hyperthermia, allowing percutaneous ablation without requiring tumor puncture. There are studies showing the efficacy of this technique in combination with TACE, with anti-tumor effect and better survival than using TACE alone $(\mathrm{Wu}, 2005)$. The method is expected to be an alternative to PEI and RFA because it avoids the puncture of the cirrhotic liver, but more studies are needed to prove its efficacy as a single therapy in the curative treatment of HCC (Maruyama et al, 2008).

Techniques for visualizing blood circulation independently from the angle of insonation based on transversal oscillation of red blood cells groups. This technique is now implemented on conventional transducers and can real time evidence different features of the blood flow visualized so far only on MRI angiography (Hansen et al, 2011).

\section{Conclusions}

Ultrasound exploration using current technologies has excellent possibilities for practical use. Several elements related to the lack of standardization and reproducibility of ultrasound procedures as well as operator-dependent nature of the method require structured approach to its use in liver tumors pathology (Bolondi et al, 2007). Structuring these applications and description of their actual performance are included in successive recommendations made by expert groups, known as "EFSUMB guidelines" (Albrecht et al, 2004; Claudon et al, 2008). In the current stage the following applications in liver pathology, especially in tumors are accepted as valid and represent indications for CEUS:

a. characterization of nodular lesions found on non-cirrhotic liver. Ultrasound examination is often the first imaging procedure performed in patients with abdominal pathology. Quite often lesions are detected and their nature has to be further on defined. Ultrasound procedures that allow achieving this purpose are multiple and 
complementary: 2D/3D ultrasound and elastography for morphometric informations; Doppler ultrasound (with its variants) and CEUS allow hemodynamic information. CEUS examination is the most specific of them as it is based on different behavior of contrast agent transiting liver masses depending on their nature. CEUS accuracy for characterization of focal lesions detected on noncirrhotic liver is similar to that of CT/MRI, being of $94.5 \%$ for metastases, $97 \%$ for hemangiomas, and of $90 \%$ for focal nodular hyperplasia. The method has a performance superior to $2 \mathrm{D}$ ultrasound and a high rate of diagnosis confidence regardless of the operator in what concerns tumor characterization and nodules count (Quaia et al, 2004).

b. characterization of nodular lesions found on the cirrhotic liver. Ultrasound examination is a "screening" procedure used to detect early HCC (Bolondi et al, 2001). The cirrhotic liver during the restructuring process develops nodules that often raise questions regarding their nature. The criteria used to determine the nature of these nodules involve size, circulation pattern (Doppler evaluated) and vascular bed behavior (CEUS evaluated). In over $90 \%$ of cases, HCC has a characteristic behavior. CEUS exploration has a sensitivity of $92-94 \%$ and a specificity of $87-96 \%$ in characterizing HCC (Tanaka et al, 2001, Nicolau et al, 2004).

In a linear study, covering 36 months, (January 2006 - December 2008), made on a cohort of 379 patients with liver tumors, (fig. 15), we had similar results concerning the performances of CEUS compared to other imaging procedures such as CT/MRI and histopathology as other groups (personal experience; unpublished results from the project Angiotumor nr. 138/2006 financed by the Ministry of Education and Research from Romania) (fig. 16) (Badea et al, 2008).

Types of liver focal lesions diagnosed by CEUS in our study

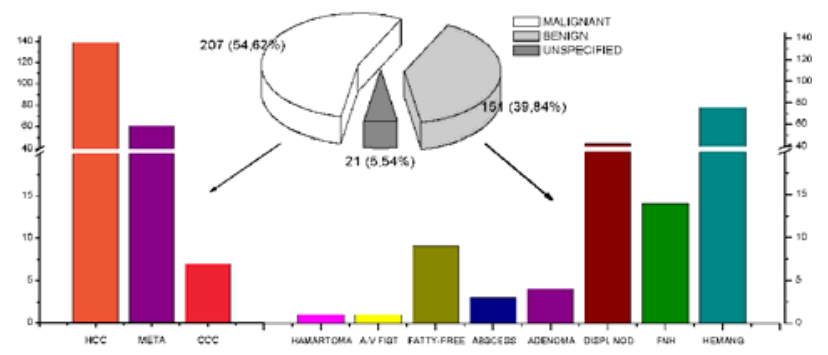

Fig. 15. Types of liver focal lesions diagnosed by CEUS in our study (HCC=hepatocellular carcinoma, META=liver metastasis, $\mathrm{CCC}=$ cholangiocarcinoma, A-V FIST=arterio-venous fistula, FATTY-FREE=fatyy-free area, DISPL NOD=cirrhotic displastic nodule, $\mathrm{FNH}=$ focal nodular hyperplasia, HEMANG=liver hemangioma)

c. Detection and characterization of liver metastases. Ultrasound is a method widely used in the early detection of liver metastases in patients with cancer pathology. Beyond the known limitations of the method, the examination is used because it is accessible, nonirradiant and it can detect metastases of quite small sizes and allows their characterization using vascular criteria. CEUS examination increases the performance of conventional ultrasound to values comparable with CT/MRI, becoming an alternative to these procedures in certain well defined situations (Dietrich et al, 2006). 


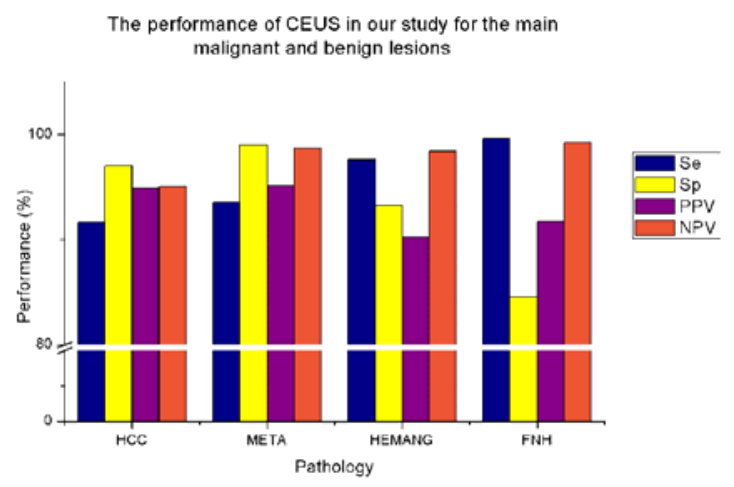

Fig. 16. The performance of CEUS in our study for the main malignant and benign lesions (HCC=hepatocellular carcinoma, META=liver metastasis, HEMANG=liver hemangioma, $\mathrm{FNH}=$ focal nodular hyperplasia, $\mathrm{Se}=$ sensitivity, $\mathrm{Sp}=$ specificity, $\mathrm{PPV}=$ positive predictive value, $N P V=$ negative predictive value)

d. Assessing therapeutic efficacy. Using the vascular criteria allows the assessment of therapeutic efficacy under chemotherapy (Bolondi et al, 2007). Performing an interventional procedure (with diagnostic or therapeutic purpose) simultaneously with the administration of i.v. CAs increases the procedure's efficiency by a better visualization of the tumor and by correct guidance of the ablation needle in the active (vascularized) area of the tumor (Skjoldbye et al, 2002, Lencioni et al, 2004). After ablative procedures, or even better in one month after the procedure, the absence of the circulation bed in the tumor in arterial phase suggests an effective treatment (Solbiati et al, 2004; Vilana et al, 2006, Bartolotta et al, 2008). This principle is also true for chemotherapy with antiangiogenic agents. Even under these conditions combination of CEUS with i.v. contrast CT examination is required to detect small metastases between procedures (Bartolotta et al, 2008).

\section{References}

Andreana L, Isgrò G, Pleguezuelo M, Germani G, Burroughs A (2009). Surveillance and diagnosis of hepatocellular carcinoma in patients with cirrhosis. World J Hepatol October 31; 1(1):48-61. ISSN 1948-5182 (online)

Badea R, Badea Gh, Galatâr N, Crăciun C, Marin M, Bucher M (1991). Aspiration of fine needle biopsy - its relevance to higher accuracy of ultrasonic diagnosis of liver tumors - investigation of 165 patients. Z Klin Med; 46 (21): 1489 - 1493

Badea R, Badea Gh (1991). Sonographische Untersuchungen des Pfortadersystems bei Lebertumoren. Ultraschall; 12 (6): 272 - 276. ISSN 0172-4614

Badea R, Socaciu M, Lupsor M, Mosteanu O, Pop T (2007). Evaluating the liver tumors using three-dimensional ultrasonography. A pictorial essay. J Gastrointestin Liv Dis; 16 (1): 85-92. ISSN 1841-8724

Badea R, Socaciu M, Caraiani C, Mosteanu O, Pop T (2008). The use of second generation Ultrasound contrast agents (SonoVue) in the diagnosis and characterization of benign liver masses. Romanian Journal of Hepatology.; 4(1):11-9. ISSN 1841-6187 
Bartolotta VT, Taibbi A, Midiri M, De Maria M 2008). Hepatocellular cancer response to radiofrequency tumor ablation: contrast-enhanced ultrasound. Abdom Imaging 33:501-511. ISSN 0942-8925 (print version) ISSN 1432-0509 (electronic version)

Bartolozzi C, Cioni D, Donati F, Granai G, Lencioni R (1999). Imaging evaluation of tumor response. In: Bartolozzi C, Lencioni R, editors. Liver malignancies. Diagnostic and interventional radiology, 1st ed. Berlin: Springer-Verlag, pp. 467-487. ISBN-10: 3540647562 ISBN-13: 9783540647560

Bolondi L, S Sofia, S Siringo, S Gaiani, A Casali, G Zironi, F Piscaglia, L Gramantieri, M Zanetti, M Sherman (2001). Surveillance programme of cirrhotic patients for early diagnosis and treatment of hepatocellular carcinoma: a cost effectiveness analysis. Gut; 48:251-259. Online ISSN 1468-3288

Bolondi L, Gaiani S, Celli N, Golfieri R, Grigioni WF, Leoni S, Venturi AM, Piscaglia F (2005). Characterization of small nodules in cirrhosis by assessment of vascularity: the problem of hypovascular hepatocellular carcinoma. Hepatology;42:27-34. Online ISSN: 1527-3350

Bolondi L, Correas JM, Lencioni R, Weskott HP, Piscaglia F (2007). New perspectives for the use of contrast-enhanced liver ultrasound in clinical practice. Digestive and Liver Disease; 39: 187-195. ISSN 1590-8658

Bruix J, Sherman M, Llovet JM, Beaugrand M, Lencioni R, Burroughs AK, Christensen E, Pagliaro L, Colombo M, Rodes J (2001). EASL Panel of Experts on HCC. Clinical management of hepatocellular carcinoma. Conclusions of the Barcelona-2000 EASL conference. European Association for the Study of the Liver. J Hepatol;35:421-430. ISSN 0168-8278

Bruix J, Sherman M (2005). Management of hepatocellular carcinoma. Hepatology;42:12081236. Online ISSN: $1527-3350$

Bruix J, Sherman M (2011) AASLD Practice Guideline. Management of Hepatocellular Carcinoma: an Update. Hepatology; 53(3): 1020-1022. Online ISSN 1527-3350

Burns PN, Wilson SR, Hope Simpson D (2000). Pulse inversion imaging of liver blood flow: an improved method for characterization of focal masses with microbubble contrast. Invest Radiol; 35:58-71. ISSN 0020-9996.

Burns PN, Wilson SR (2006). Microbubble contrast for radiological imaging: 1. Principles. Ultrasound Q;22(1):5-13. PMID: 16641788

Cherrington JM, Strawn LM, Shawver LK (2000). New paradigms for the treatment of cancer: the role of anti-angiogenesis agents. Adv Cancer Res;79:1-38. ISSN 0065-230X

Choudhry S, Gorman B, Charboneau JW, Tradup JD, Beck RJ, Kofler JM, Groth DS (2000). Comparison of tissue harmonic imaging with conventional US in abdominal disease. Radiographics;20: 1127 - 1135. Print ISSN 0271-5333 Online ISSN 1527-1323

Claudon M, Cosgrove D, Albrecht T, Bolondi L, Bosio M, Calliada F, Correas JM, Darge K, Dietrich C, D'Onofrio M, Evans DH, Filice C, Greiner L, Jäger K, Jong N, Leen E, Lencioni R, Lindsell D, Martegani A, Meairs S, Nolsøe C, Piscaglia F, Ricci P, Seidel G, Skjoldbye B, Solbiati L, Thorelius L, Tranquart F, Weskott HP, Whittingham T (2008). Guidelines and good clinical practice recommendations for contrast enhanced ultrasound (CEUS)-update 2008. Ultraschall Med; 29: 28-44. ISSN 01724614.

Cosgrove D, Eckersley R (1997). Doppler indices in tumors - resolution of a dilemma? Ultrasound in Obstetrics and Gynecology, 10: 9 - 11. ISSN 1469-0705

Cosgrove D, Blomley M (2004). Liver tumors: evaluation with contrast-enhanced ultrasound. Abdom Imaging; 29: 446-454. ISSN: 0942-8925 (print version) ISSN 14320509 (electronic version) 
Derchi LE, Claudon M (2009). Ultrasound: a strategic issue for radiology? Eur Radiol; 19: 16. Published online 15 August 2008. ISSN 0938-7994 (print version) ISSN 1432-1084 (electronic version)

Dietrich CF, Kratzer W, Strobe D, Danse E, Fessl R, Bunk A, Vossas U, Hauenstein K, Koch W, Blank W, Oudkerk M, Hahn D, Greis C. (2006). Assessment of metastatic liver disease in patients with primary extra-hepatic tumors by contrast-enhanced sonography versus CT and MRI. World J Gastroenterol;12:1699-1705. ISSN 1007-9327 (print) ISSN 2219-2840 (online)

Dromain C, de Baere T, Elias D, Kuoch V, Ducreux M, Boige V, Petrow P, Roche A, Sigal R (2002). Hepatic tumors treated with percutaneous radio-frequency ablation: $\mathrm{CT}$ and MR imaging follow up. Radiology; 223: 255-262. Print ISSN 0033-8419. Online ISSN 1527-1315

Ewertsen C, Henriksen B. M, Torp-Pedersen S, Bachmann Nielsen M (2011). Characterization by Biopsy or CEUS of Liver Lesions Guided by Image Fusion between Ultrasonography and CT, PET/CT or MRI. Published online January 11, 2011 Ultraschall in Med; 32: 191-197. ISSN 0172-4614.

Frieser M, Kiesel J, Lindner A, Bernatik T, Haensler J. M., Janka R, Hahn E. G., Strobel D. (2011). Efficacy of Contrast-Enhanced US versus CT or MRI for the Therapeutic Control of Percutaneous Radiofrequency Ablation in the Case of Hepatic Malignancies. Ultraschall in Med. Published online 2011. ISSN 0172-4614.

Gaiani S, Volpe L, Piscaglia F, Bolondi L (2001). Vascularity of liver tumours and recent advances in Doppler ultrasound. J Hepatol;34:474-482. ISSN 0168-8278

Hansen PM, Pedersen MM., Kristoffer, Hansen KL., Bachmann Nielsen M, Jensen JA (2011). Demonstration of a Vector Velocity Technique. Ultraschall in Med; 32:213-215. ISSN $0172-4614$.

Horigome H, Nomura T, Saso K, Itoh M, Joh T, Ohara H (1999). Limitations of imaging diagnosis for small hepatocellular carcinoma: comparison with histological findings. J Gastroenterol Hepatol; 14: 559 -565. Online ISSN 1440-1746

International Working Party (1995). Terminology of nodular hepatocellular lesions. Hepatology;22:983-993. Online ISSN 1527-3350

Jang HJ, Kim TK, Wilson SR (2009). Small nodules $(1-2 \mathrm{~cm})$ in liver cirrhosis: characterization with contrast-enhanced ultrasound. Eur J Radiol; 72: 418-424. ISSN 0720-048X

Kojiro M (2004). Focus on dysplastic nodules and early hepatocellular carcinoma: an Eastern point of view. Liver Transpl;10:S3-S8. Online ISSN 1527-6473

Konopke R, Kersting S, Saeger HD, Bunk A (2005). Detection of liver lesions by contrastenhanced ultrasound -- comparison to intraoperative findings. Ultraschall in Med; 26: 107-113. ISSN 0172-4614.

Kreft B, Pauleit D, Bachmann R, Conrad R, Krämer A, Schild HH (2001). Incidence and significance of small focal liver lesions in MRI. Rofo; 173: 424-429. PubMed: 11414150. Available from www.ncbi.nlm.nih.gov

Larsen LPS (2010). Role of contrast enhanced ultrasonography in the assessment of hepatic metastases: A review. World J Hepatol January 27; 2(1): 8-15. ISSN 1948-5182 (online)

Lassau N, Chami L, Chebil M, Benatsou B, Bidault S, Girard E, Abboud G, Roche A (2011). Dynamic Contrast-Enhanced Ultrasonography (DCE-US) and Anti-angiogenic Treatments. Discovery Medicine. Published online. ISSN 1539-6509; eISSN 1944-7930

Lencioni R, Crocetti L, Cioni D, Della Pina C, Bartolozzi C (2004). Percutaneous radiofrequency ablation of hepatic colorectal metastases. Technique, indications, results, and new promises. Invest Radiol;39:689-697. ISSN 0020-9996 
Lencioni R, Piscaglia F, Bolondi L (2008). Contrast-enhanced ultrasound in the diagnosis of hepatocellular carcinoma. Journal of Hepatology 48 848-857. ISSN 0168-8278

Lim HS, Jeong YY, Kang HK, Kim JK, Park JG (2006). Imaging features of hepatocellular carcinoma after transcatheter arterial chemoembolization and radiofrequency ablation. Am J Roentgenol; 187: W341-W349. Print ISSN: 0361-803X Online ISSN: 1546-3141

Lindner J (2004). Microbubbles in medical imaging: current applications and future directions. Nature;3:527-532. ISSN 0028-0836, eISSN: 1476-4687

Llovet JM, Bru'C, Bruix J (1999). Prognosis of hepatocellular carcinoma: the BCLC staging classification. Semin Liver Dis;19:329-338. Print ISSN 0272-8087, e ISSN 1098-8971

Llovet JM, Burroughs A, Bruix J (2003). Hepatocellular carcinoma. Lancet;362:1907-1917. Print ISSN 0140-6736.

Llovet JM, Bruix J (2008). Novel advancements in the management of hepatocelular carcinoma in 2008. Journal of Hepatology; 48: S20 - S37. ISSN 0168-8278

Luo W, Numata K, Morimoto M, Kondo M, Takebayashi S, Okada M, Morita S, Tanaka K (2009). Focal liver tumors: characterization with 3D perflubutane microbubble contrast agent-enhanced US versus 3D contrast-enhanced multidetector CT. Radiology; 251(1):287-95. Print ISSN 0033-8419. Online ISSN 1527-1315

Maruyama H, Yoshikawa M, Yokosuka O (2008). Current role of ultrasound for the management of hepatocellular carcinoma. World J Gastroenterol; 14: 1710-1719. ISSN 1007-9327

Matsui O (2004). Imaging of multistep human hepatocarcinogenesis by CT during intraarterial contrast injection. Intervirology; 47: 271-276. print ISSN 0300-5526, eISSN 1423-0100

Minami Y, Kudo M (2010). Hepatic malignancies: Correlation between sonographic findings and pathological features. World J Radiol 28;2(7):249-256. ISSN 1949-8470 (online)

Molins IG, Fernández Font JM, Álvaro JC, Navarro JLL, Gil MF, Rodríguez CMF (2010). Contrast-enhanced ultrasound in diagnosis and characterization of focal hepatic lesions. World J Radiol 2010 December 28; 2(12): 455-462. ISSN 1949-8470 (online)

Newman \& T Bettinger (2007). Gene therapy progress and prospects: Ultrasound for gene transfer. Gene Therapy 14, 465-475. ISSN 0969-7128, eISSN 1476-5462

Nicolau C, Catala V, Vilana R (2004). Evaluation of hepatocellular carcinoma using Sonovue, a second generation ultrasound contrast agent: Correlation with cellular differentiation. Eur Radiol;14:1092-1099. print ISSN 0938-7994, eISSN 1432-1084

Nicolau C, Vilana R, Bianchi L, Brú C (2007). Early-stage hepatocellular carcinoma: the high accuracy of real-time contrast-enhanced ultrasonography in the assessment of response to percutaneous treatment. Eur Radiol; 17(Suppl 6): F80-88. print ISSN 0938-7994, eISSN: 1432-1084

Parkin DM, Bray F, Ferlay J, Pisani P (2005). Global cancer statistics 2002. CA Cancer J Clin;55:74-108. Print ISSN: 0007-9235 eISSN: 1542-4863

Piscaglia F, Corradi F, Mancini M, Giangregorio F, Tamberi S, Ugolini G, Cola B, Bazzocchi A, Righini R, Pini P, Fornari F, Bolondi L (2007). Real time contrast enhanced ultrasonography in detection of liver metastases from gastrointestinal cancer. BMC Cancer; 7: 171. ISSN 1471-2407

Quaia E, Calliada F, Bertolotto M, Rossi S, Garioni L, Rosa L, Pozzi-Mucelli R. (2004) Characterization of focal liver lesions with contrast- specific US modes and a sulfur hexafluoride-filled microbubble contrast agent: diagnostic performance and confidence. Radiology;232(2): 420-30. Print ISSN 0033-8419. Online ISSN 1527-1315 
Quaia E, Palumbo A, Rossi S, Degobbis F, Cernic S, Tona G, Cova M (2006). Comparison of Visual and Quantitative Analysis for Characterization of Insonated Liver Tumors After Microbubble Contrast Injection. AJR; 186:1560-1570. ISSN 1007-9327

Sandulescu DL, Dumitrescu D, Rogoveanu I, Saftoiu A (2011). Hybrid ultrasound imaging techniques (fusion imaging). World J Gastroenterol; 17(1):49-52. ISSN 1007-9327 (print) ISSN 2219-2840 (online)

Skjoldbye B, Pedersen MH, Struckmann J, Burcharth F, Larsen T (2002). Improved detection and biopsy of solid liver lesions using pulse-inversion ultrasound scanning and contrast agent infusion. Ultrasound Med Biol;28:439-444. ISSN 0301-5629

Solbiati L, Ierace T, Tonolini M, Cova L (2004). Guidance and monitoring of radiofrequency liver tumor ablation with contrast-enhanced ultrasound. Eur J Radiol; 51(Suppl): S19-23. print ISSN 0938-7994 eISSN 1432-1084

Solbiati L, Tonolini M, Cova L (2004). Monitoring RF ablation. Eur Radiol;14:34-42. print ISSN 0938-7994 eISSN 1432-1084

Spârchez Z, Radu P, Anton O, Socaciu M, Badea R (2009). Contrast-Enhanced Ultrasound in Assessing Therapeutic Response in Ablative Treatments of Hepatocellular Carcinoma. J Gastrointestin Liver Dis Vol.18 No 2, 243-248. ISSN 1841-8724

Strobel D, Kleinecke C, Hänsler J, Frieser M, Händl T, Hahn EG, Bernatik T (2005). Contrastenhanced sonography for the characterisation of hepatocellular carcinomascorrelation with histological differentiation. Ultraschall in Med;26:270-276. ISSN 0172-4614.

Tanaka S, Ioka T, Oshikawa O, Hamada Y, Yoshioka F (2001). Dynamic sonog- raphy of hepatic tumors. Am J Roentgenol;177:799-805. Online ISSN 1546-3141

Vilana R, Bianchi L, Varela M, Nicolau C, Sánchez M, Ayuso C, García M, Sala M, Llovet JM, Bruix J, Bru C; BCLC Group (2006). Is microbubble-enhanced ultrasonography sufficient for assessment of response to percutaneous treatment in patients with early hepatocellular carcinoma? Eur Radiol;16:2454-2462. print ISSN 0938-7994 eISSN 1432-1084

von Herbay A, Vogt C, Westendorff J, Häussinger D, Gregor M (2009). Correlation between SonoVue Enhancement in CEUS, HCC Differentiation and HCC Diameter: Analysis of 130 Patients with Hepatocellular Carcinoma (HCC). Ultraschall in Med; 30: 544550. ISSN 0172-4614.

Weidener N, Semple JP, Welch WR, Folkman J (1991). Tumor angiogenesis and metastasis correlation in invasive breast carcinoma. N. Engl. J. Med, 324, 1 - 7. Available from: http:/ / www.nejm.org/doi/full/10.1056/NEJM199101033240101

Wernecke K, Rummeny E, Bongartz G, Vassallo P, Kivelitz D, Wiesmann W, Peters PE, Reers B, Reiser M, Pircher W (1991). Detection of hepatic masses in patients with carcinoma: comparative sensitivities of sonography, CT, and MR imaging. Am J Roentgenol; 157: 731-739. Online ISSN 1546-3141

Wu F, Wang ZB, Chen WZ, Zou JZ, Bai J, Zhu H, Li KQ, Jin CB, Xie FL, Su HB (2005). Advanced hepatocellular carcinoma: treatment with high-intensity focused ultrasound ablation combined with transcatheter arterial embolization. Radiology; 235: 659-667. Print ISSN 0033-8419. Online ISSN 1527-1315

Zapata E, Zubiaurre L, Castiella A, Salvador P, García-Bengoechea M, Esandi P, Arriola A, Beguiristain A, Ruiz I, Garmendia G, Orcolaga R, Alustiza JM. (2010). Are hepatocellular carcinoma surveillance programs effective at improving the therapeutic options? Rev Esp Enferm Dig; 102: 484-488. ISSN 1130-0108 


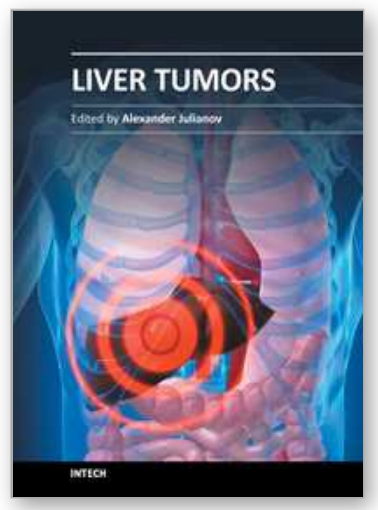

\author{
Liver Tumors \\ Edited by Prof. Alexander Julianov
}

ISBN 978-953-51-0036-2

Hard cover, 200 pages

Publisher InTech

Published online 03, February, 2012

Published in print edition February, 2012

This book is oriented towards clinicians and scientists in the field of the management of patients with liver tumors. As many unresolved problems regarding primary and metastatic liver cancer still await investigation, I hope this book can serve as a tiny step on a long way that we need to run on the battlefield of liver tumors.

\title{
How to reference
}

In order to correctly reference this scholarly work, feel free to copy and paste the following:

R. Badea and Simona loanitescu (2012). Ultrasound Imaging of Liver Tumors - Current Clinical Applications, Liver Tumors, Prof. Alexander Julianov (Ed.), ISBN: 978-953-51-0036-2, InTech, Available from:

http://www.intechopen.com/books/liver-tumors/ultrasound-imaging-of-liver-tumors-current-clinical-applications

\section{INTECH}

open science | open minds

\section{InTech Europe}

University Campus STeP Ri

Slavka Krautzeka 83/A

51000 Rijeka, Croatia

Phone: +385 (51) 770447

Fax: +385 (51) 686166

www.intechopen.com

\section{InTech China}

Unit 405, Office Block, Hotel Equatorial Shanghai

No.65, Yan An Road (West), Shanghai, 200040, China

中国上海市延安西路65号上海国际贵都大饭店办公楼405单元

Phone: +86-21-62489820

Fax: +86-21-62489821 
(C) 2012 The Author(s). Licensee IntechOpen. This is an open access article distributed under the terms of the Creative Commons Attribution 3.0 License, which permits unrestricted use, distribution, and reproduction in any medium, provided the original work is properly cited. 